\title{
NOTAS SOBRE LA EVOLUCION Y ESTRUCTURA DEL TRANSPORTE EN ESPAÑA Y SU INCIDENCIA EN EL DESARROLlo REGIONAL
}

656: $353: 338.984 .3$

\author{
por \\ Ignacio Ballester Ros
}

SUMARIO: I. INTRODUCCION.-II. EVOLUCION DEL TRANSPORTE TERRESTRE EN ESPAÑA.-III. EL TRAFICO DE MERCANCIAS POR CARRETERA Y POR FERROCARRIL.-IV. EL TRANSPORTE DE VIAJEROS.-V. INCIDENCIA DEL TRANSPORTE EN EL DESARROLlO REGIONAL: 1. Principales Realizaciones. 2. ConcluSIONES.

\section{INTRODUCCION}

Bajo el patrocinio de la Subsecretaría de Planificación de la Presidencia del Gobierno se ha publicado en el mes de julio de 1977 un estudio elaborado por un equipo de economistas $e$ ingenieros sobre la "Incidencia del transporte en el desarrollo regional».

El estudio comprende cinco partes, precedidas de una amplia información. En esta última se fijan su objetivo y alcance, así como las conclusiones que del mismo se deducen.

En la primera parte se expone la teoría del transporte y el desarrollo, un estudio empírico sobre el papel del transporte en el desarrollo regional de los Estados Unidos y las experiencias más recientes, con detalle de la planificación regional en varios países europeos. Igualmente se analizan las tendencias de la política de 
transporte-desarrollo regional en España a través de los Planes de Desarrollo y los criterios aplicados y el papel desempeñado por las empresas públicas y privadas en la localización de las inversiones y desinversiones del sector transporte.

La segunda parte se dedica al examen de la evolución histórica del transporte terrestre en España, sus condicionamientos geográficos, sus alternativas técnicas e institucionales, el impacto regional y su influencia sobre los asentamientos poblacionales.

En la tercera parte se describe la estructura del tráfico terrestre en España, se analizan el tráfico por carretera y por ferrocarril y la competencia entre ambos, con sus características y efectos.

La cuarta parte comprende una descripción del modelo aplicado para el estudio del transporte y desarrollo regional en España.

Y en la quinta se aborda el caso de Galicia, con el esquema histórico de sus comunicaciones, la estructura de su tráfico, la aplicación del modelo de gravedad al de mercancías, la situación actual y las proyecciones futuras de tráfico.

El objetivo del estudio es el «análisis y evolución del impacto físico del transporte sobre el territorio en cuanto a su utilización y revalorización del suelo, localización industrial y habitacional y determinación de los tipos de beneficios y costes económicos que podrían derivarse de las inversiones y su importancia relativa», tratando, en una primera etapa, de formar un «inventario de objetivos coordinados del transporte y desarrollo regional para las grandes áreas regionales, definiendo los grandes corredores de comunicación terrestre de carga y pasajeros, la concentración o desconcentración urbana, la política migratoria y la limitación de conflictos ecológicos en dichas áreas» y de definir las variables económicas más importantes en la ejecución de la política nacional del transporte.

La importancia de este estudio y su interés en el ámbito local hace aconsejable que se le dedique una crónica, al objeto de resaltar las características estructurales y coyunturales del fenómeno del transporte, sector básico en la economía nacional, y, singularmente, en el planeamiento urbano y en el desarrollo regional. 


\section{EVOLUCION DEL TRANSPORTE TERRESTRE EN ESPAÑA}

Las condiciones geográficas y demográficas de España no son favorables para un adecuado sistema de transporte, ya que se trata de un país extenso, con baja densidad de población, con los centros poblacionales más importantes muy separados entre sí y con un tráfico fluvial de mercancías prácticamente nulo.

La irregular orografía peninsular no permitió las relaciones comerciales a lo largo de los valles, siguiendo el cauce de los ríos, como en Europa, y hubo que potenciar los pasos de montaña, únicos nexos entre grandes valles.

Las lineas naturales de comunicación se establecen, durante largo tiempo, de norte a sur, dividiendo a la península en cuatro corredores de tráfico:

1. Galicia-Portugal, por la llanura litoral atlántica.

2. Asturias-León-Extremadura-Andalucía occidental, a través del puerto de Pajares y la brecha del Alagón.

3. Santander-Castilla la Vieja-Castilla la Nueva-Andalucía, por los pasos de Reinosa, Guadarrama y Despeñaperros.

4. Cataluña-Valencia-Murcia, por la costa mediterránea.

Los corredores segundo y tercero tienen núcleos de relación entre Castilla y León, y los corredores tercero y cuarto se unen a través de Aragón, que enlaza a Castilla con Cataluña por la brecha del Jalón y los pasos de Fraga.

La primera manifestación histórica del aprovechamiento de pasos del Norte hacia el Sur son las rutas de la trashumancia institucionalizadas desde el siglo XI a través de las cañadas y veredas.

Felipe V dicta en 1718 una ordenanza sobre construcción de caminos, mediante una red vial, de disposición radial, con centro en Madrid, y Carlos III, por otra ordenanza de 10 de junio de 1847, ordena el remozamiento de la red de Madrid a Cataluña, Valencia, Andalucía y Galicia. Este sistema potencia a Madrid como centro de actividad económica, política y de consumo y debilita la «Ruta de la Plata». De 1800 a 1868 se construyen $7.822 \mathrm{~km}$. de carreteras, política que no se interrumpe con la introducción del ferrocarril.

El transporte interior de mercancías se atendía con animales de carga y carros, y desde mediados del siglo xvirI, con diligencias para 
pasajeros. Este sistema no puede atender, con su carácter estacionario, la creciente demanda de transporte por parte de la Corte y del Ejército.

Hasta el año 1800, a excepción de Madrid, las demás ciudades que superaban los 50.000 habitantes eran puertos marítimos. Sólo Madrid y Barcelona superaban los 100.000 habitantes, y Valencia, Sevilla, Cádiz y Málaga, los 50.000. En el interior apenas existen otras aglomeraciones urbanas capaces de generar actividad con incidencia regional y fomentar mercados de cierta entidad.

El establecimiento de un adecuado sistema de transporte se retrasa por las crisis estacionales y las guerras napoleónicas y coloniales.

Concebido el sistema de canales por Carlos I y Felipe II, sólo se concretó en la construcción de algunos tramos del Canal de Aragón y del Canal de Castilla, fracasando los planes más ambiciosos, porque España no dispone de amplios valles y ríos tan caudalosos como los europeos.

Nace entonces la esperanza en el ferrocarril, iniciándose su construcción con medio siglo de retraso respecto a Europa. BarcelonaMataró en 1848, Madrid-Aranjuez en 1851, Sama de Langreo-Gijón en 1856 y El Grao de Valencia-Játiva-Almansa son los primeros tramos construidos. Los obstáculos geográficos, la inestabilidad política y la falta de capitales dificultan su desarrollo. En general, la red ferroviaria, concebida con un trazado en forma radial, como la de carreteras, sin apenas tramos periféricos, es obra de compañías privadas, con participación de capital extranjero y subvenciones del Estado en algunos casos. En 1885 se explotaban $8.933 \mathrm{~km}$. y se hallaban en construcción 3.660. A partir de 1888 se inicia la construcción de ferrocarriles de vía estrecha. A principios del siglo $\mathrm{xx}$ la red está prácticamente terminada y dispone de $14.000 \mathrm{~km}$. de vías, de los que el 80 por 100 son de vía estrecha. En la década de 1910 se construyen $2.500 \mathrm{~km}$. más.

Las compañías privadas llegan al límite de sus posibilidades al estancarse el comercio europeo con motivo de la primera guerra mundial. El Estatuto ferroviario trata de paliar sus efecto. En 1941 se crea la Red Nacional de los Ferrocarriles Españoles (RENFE) con todas las líneas de vía ancha, cuando la red supera los 16.500 kilómetros, y se pasa del régimen de concesiones al de nacionalización. Con el Plan de Modernización de la RENFE de 1960 el Estado 
se hace cargo de la infraestructura y de la mejora de los servicios y se terminan nuevas líneas: Madrid-Burgos y El Ferrol-Gijón.

El: ferrocarril, con una explotación deficitaria, se pone a la defensiva frente a la carretera, que ha absorbido un tráfico, muchas veces inadecuado, y nace la competencia entre ambos medios de transporte.

\section{EL TRAFICO DE MERCANCIAS POR CARRETERA Y POR FERROCARRIL}

El estudio del transporte por carretera y por ferrocarril debe realizarse teniendo en cuenta, de una parte, su impacto espacial dentro y fuera de las Provincias y Regiones, y de otra, los costes respectivos, distinguiendo el tráfico de mercancías y de pasajeros por carretera y por ferrocarril y examinando la competencia carretera-ferrocaril y sus efectos respecto a la ordenación del transporte.

Para el estudio del tráfico de mercancias por carretera se dispone de los resultados sobre la capacidad de carga y de las matrices origen-destino obtenidos en la "I Encuesta Nacional de Transporte de Mercancías por Carretera", realizada en 1969 por el Instituto Nacional de Estadística, que se presentan, por Provincias, en la siguiente tabla:

\begin{tabular}{|c|c|c|c|}
\hline \multirow{2}{*}{ Provincias } & \multicolumn{3}{|c|}{ TRAFICO (MILES DE TONELADAS) } \\
\hline & Originadas & Destinadas & Diferencias \\
\hline 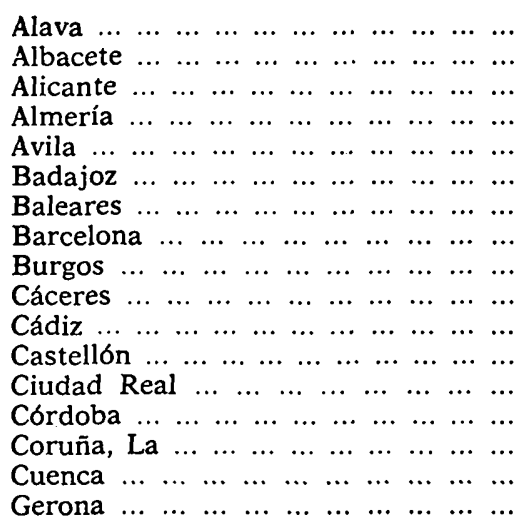 & $\begin{array}{r}4.309,6 \\
3.866,7 \\
9.979,0 \\
4.231,4 \\
1.312,3 \\
6.159,2 \\
8.885,0 \\
60.979,5 \\
5.771,5 \\
6.764,3 \\
9.241,1 \\
5.362,0 \\
4.612,1 \\
10.470,0 \\
10.949,1 \\
3.310,9 \\
11.189,1\end{array}$ & $\begin{array}{r}4.959,2 \\
3.675,9 \\
10.153,4 \\
4.230,5 \\
1.175,7 \\
6.276,4 \\
8.885,0 \\
63.253,6 \\
5.574,4 \\
6.945,1 \\
10.066,0 \\
4.915,9 \\
4.308,6 \\
10.473,3 \\
10.968,1 \\
3.062,9 \\
11.629,2\end{array}$ & $\begin{array}{r}649,6 \\
190,8 \\
-174,4 \\
0,9 \\
136,6 \\
-\quad 117,2 \\
-2.274,1 \\
197,1 \\
-\quad 180,7 \\
-\quad 824,9 \\
446,1 \\
303,5 \\
-\quad 3,3 \\
-\quad 19,0 \\
-\quad 448,0 \\
440,1\end{array}$ \\
\hline
\end{tabular}




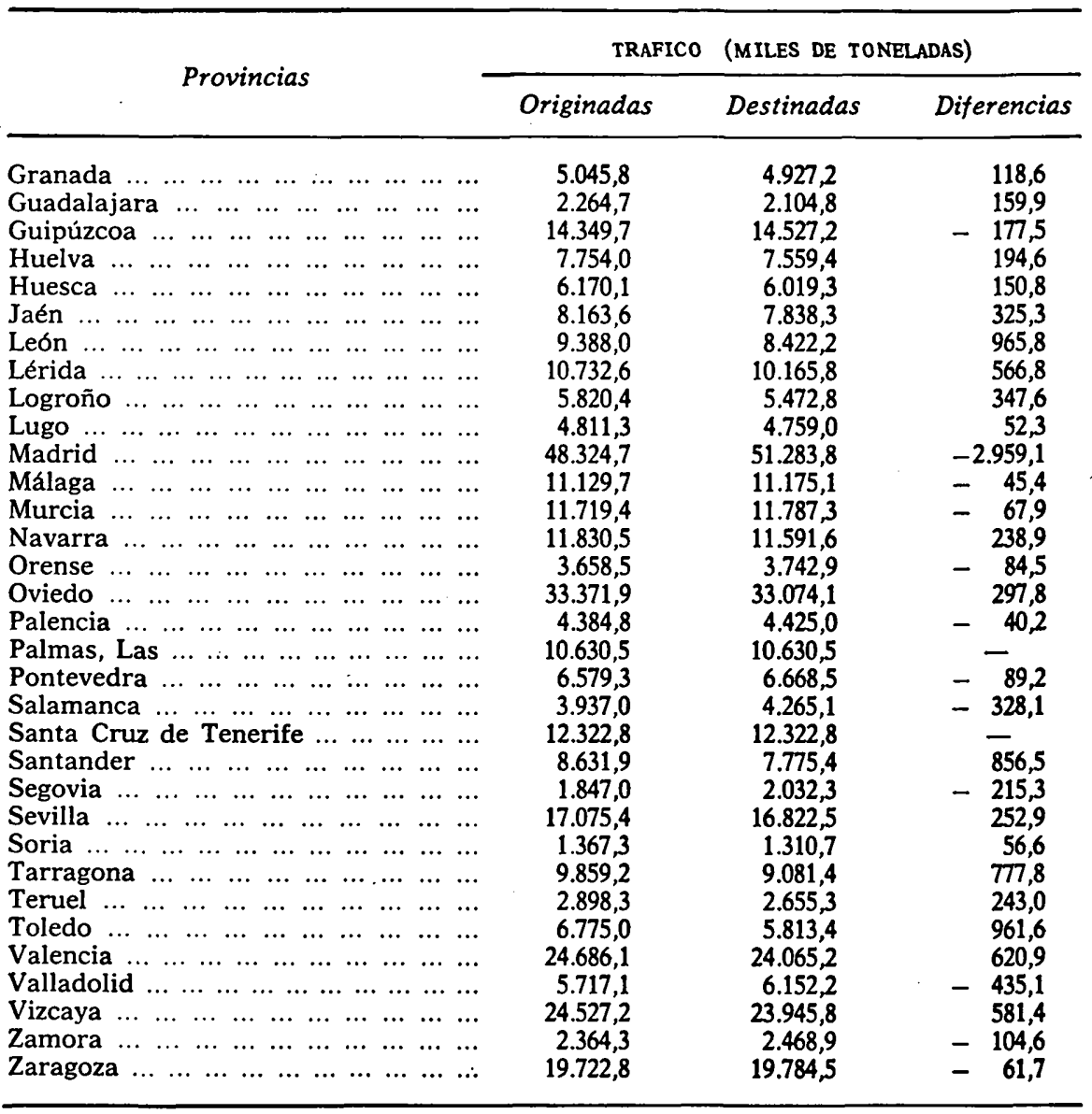

Las diferencias o saldos, positivos o negativos, entre las cifras de toneladas originadas o destinadas permiten clasificar a las Provincias en exportadoras o importadoras, figurando 26 entre las primeras y 21 entre las últimas.

En general, destacan entre las exportadoras Provincias de economía predominantemente agraria, en ambas Mesetas, Valle del Ebro, Andalucía y litoral mediterráneo; algunas, en la orla cantábrica, de actividad industrial preponderante que se abastecen de materias primas en la Provincias o por mar o que envían semielaboradas a otras Provincias, y otras, como Valencia, de economía mixta, agrícola e industrial.

Las Provincias importadoras destacan, en general, por su actividad industrial o de servicios, que se han de abastecer de materias 
primas fuera de la Provincia y atender a las necesidades de importantes concentraciones urbanas. Entre ellas aparecen, por excepción, varias Provincias agrícolas de Galicia, Valle del Duero y Extremadura. Para comprender esta aparente anomalía conviene subrayar que las cifras comentadas se refieren al tráfico por carretera y no al tráfico total.

Un segundo aspecto de las matrices origen-destino es la relación existente entre el tráfico interior de cada Provincia -intraprovincial- y el tráfico total de la misma, que se recoge en esta tabla:

TRAFICO INTRAPROVINCIAL

\begin{tabular}{ccc}
\hline & Porcentaje & Porcentaje \\
Provincias & de este trafi- de este traff- \\
Miles de & co sobre el co sobre el \\
toneladas & volumen total volumen total \\
de toneladas de toneladas \\
originadas en destinadas a \\
cada Provincia cada Provincia
\end{tabular}

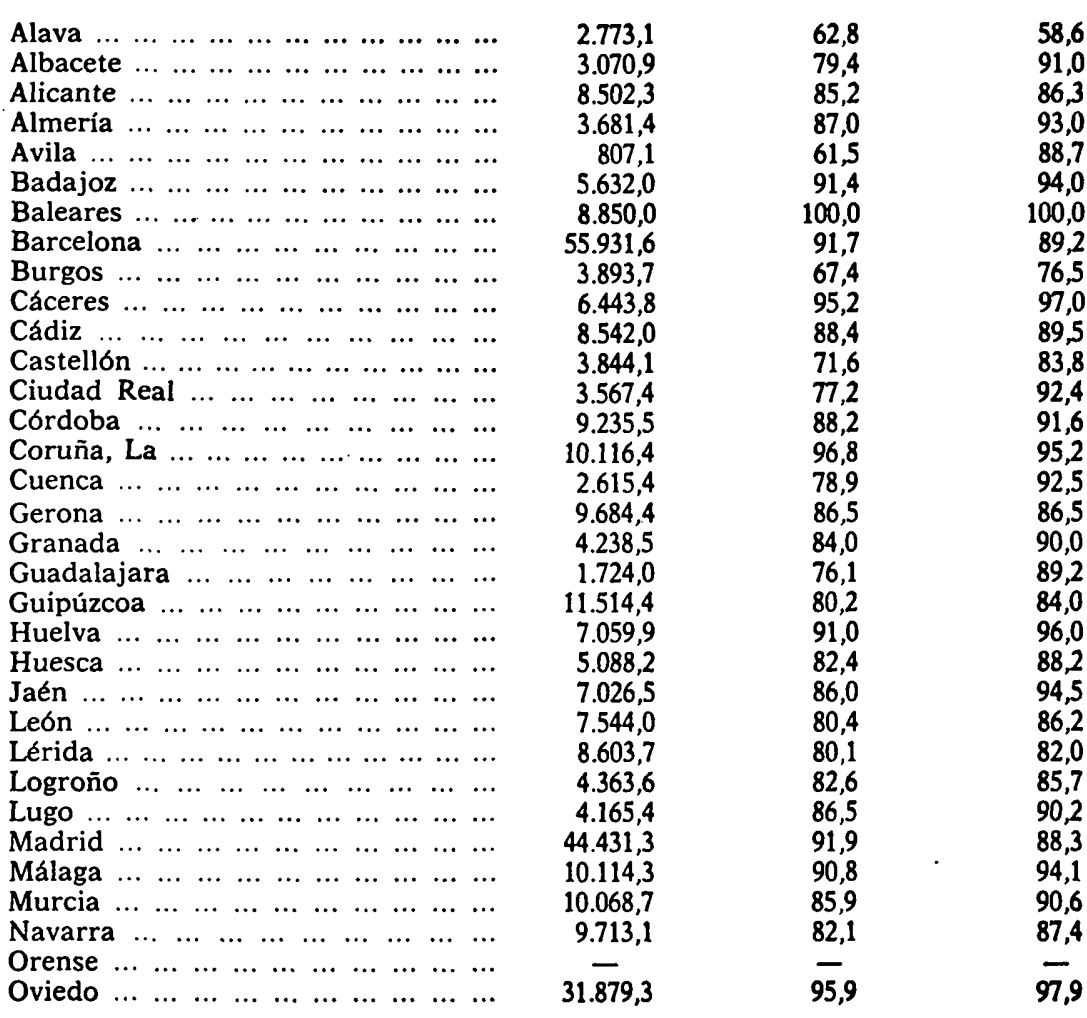




\begin{tabular}{|c|c|c|c|}
\hline \multirow[b]{2}{*}{ Provincias } & \multicolumn{3}{|c|}{ TRAFICO INTRAPROVINCIAL } \\
\hline & $\begin{array}{l}\text { Miles de } \\
\text { toneladas }\end{array}$ & $\begin{array}{l}\text { Porcentaje } \\
\text { de este trdfi- } \\
\text { co sobre el } \\
\text { volumen total } \\
\text { de toneladas } \\
\text { originadas en } \\
\text { cada Provincia }\end{array}$ & $\begin{array}{l}\text { Porcentaje } \\
\text { de este trafi- } \\
\text { co sobre el } \\
\text { volumen total } \\
\text { de toneladas } \\
\text { destinadas a } \\
\text { cada Provincia }\end{array}$ \\
\hline 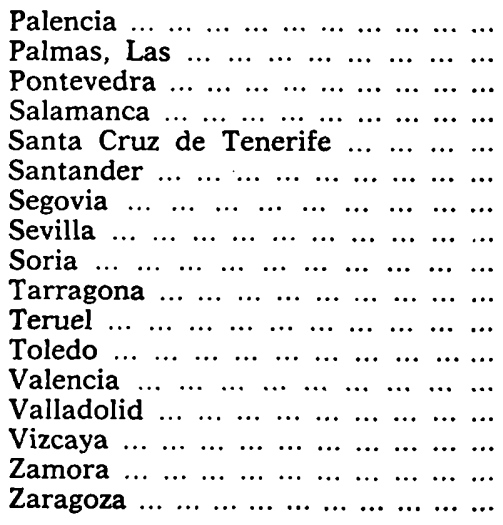 & $\begin{array}{r}3.386,9 \\
10.630,5 \\
5.732,9 \\
3.503,2 \\
12.322,8 \\
6.523,2 \\
1.453,6 \\
14.666,8 \\
1.141,5 \\
7.460,2 \\
2.242,0 \\
4.077,3 \\
20.438,0 \\
4.632,0 \\
20.850,4 \\
2.067,0 \\
17.501,4\end{array}$ & $\begin{array}{r}77,2 \\
100,0 \\
87,1 \\
88,9 \\
100,0 \\
75,5 \\
79,9 \\
85,8 \\
83,4 \\
75,6 \\
77,3 \\
60,1 \\
82,7 \\
81,0 \\
85,0 \\
\overline{88,7}\end{array}$ & $\begin{array}{r}81,0 \\
100,0 \\
90,0 \\
94,5 \\
100,0 \\
90,0 \\
81,5 \\
85,5 \\
100,0 \\
84,0 \\
91,5 \\
88,0 \\
87,6 \\
85,4 \\
89,7 \\
91,2 \\
88,4\end{array}$ \\
\hline
\end{tabular}

Los porcentajes son altos, en todo caso, porque el transporte por carretera es el idóneo para distancias cortas dentro de una misma Provincia. La primera columna es un indicador del autoconsumo relativo de cada Provincia, y la segunda lo es del nivel de autoabastecimiento.

Los factores influyentes en este tráfico son básicamente: la extensión de la Provincia en cuestión, que se refleja en los valores altos para Extremadura, y los relativamente bajos para las Vascongadas; la importancia relativa de un determinado sector económico, como el de servicios en Madrid o Málaga; la existencia de puertos importantes por los que se canalice la exportación provincial, como Barcelona, Gijón o Huelva, y, finalmente, el nivel de especialización económica de la Provincia, ya que las subdesarrolladas y agrarias no son susceptibles de originar tráfico «intraprovincial» importante, $\mathrm{y}$, por el contrario, las más especializadas en la producción de determinados bienes originan un elevado tráfico dirigido hacia otras Provincias, que hace disminuir su porcentaje de tráfico intraprovincial. 
Para el análisis del tráfico inter $e$ intrarregional se ha considerado dividida a la península en ocho grandes Regiones $\left(^{*}\right)$. De las submatrices origen-destino, elaboradas para cada una de las Regiones consideradas que incluyen en cada caso la Región en cuestión y la más próxima o las dos más próximas, se ha deducido la siguiente tabla:

\begin{tabular}{|c|c|c|c|c|c|c|c|c|}
\hline & \multirow{2}{*}{\multicolumn{2}{|c|}{ Regiones }} & & \multicolumn{2}{|c|}{$\begin{array}{c}\text { Porcentaje del trafico } \\
\text { intrarregional sobre } \\
\text { el total }\end{array}$} & \multicolumn{2}{|c|}{$\begin{array}{l}\text { Porcentaje del trafico } \\
\text { interprovincial de la } \\
\text { Región considerada } \\
\text { más la Región más } \\
\text { proxima y Madrid } \\
\text { sobre el tráfico } \\
\text { interprovincial total }\end{array}$} & \multirow[t]{2}{*}{$\begin{array}{l}\text { Región } \\
\text { conside- } \\
\text { rada }\end{array}$} \\
\hline & & & & Originado & Destinado & Originado & Destinado & \\
\hline $\begin{array}{r}\text { I } \\
\text { II } \\
\text { III } \\
\text { IV } \\
\text { V } \\
\text { VI } \\
\text { VII }\end{array}$ & $\begin{array}{lll}\ldots & \cdots & \cdots \\
\ldots & \cdots & \cdots \\
\ldots & \cdots & \cdots \\
\ldots & \cdots & \cdots \\
\ldots & \cdots & \cdots \\
\ldots & \cdots & \cdots \\
\ldots & \cdots & \cdots\end{array}$ & $\begin{array}{l}\cdots \\
\cdots \\
\cdots \\
\cdots \\
\cdots \\
\cdots \\
\cdots\end{array}$ & $\begin{array}{l}\cdots \\
\cdots \\
\cdots \\
\cdots \\
\cdots \\
\cdots\end{array}$ & $\begin{array}{r}59,1 \\
32,3 \\
18,3 \\
46,3 \\
52,3 \\
9,4 \\
90,6\end{array}$ & $\begin{array}{l}57,3 \\
34,7 \\
20,7 \\
43,3 \\
49,7 \\
15,1 \\
55,5\end{array}$ & $\begin{array}{l}73,8 \\
45,9 \\
49,0 \\
65,7 \\
58,1 \\
28,9 \\
58,5\end{array}$ & $\begin{array}{l}67,7 \\
44,4 \\
46,8 \\
61,5 \\
63,3 \\
25,8 \\
59,7\end{array}$ & $\begin{array}{c}\text { II } \\
\text { III } \\
\text { IV } \\
\text { III } \\
\text { VI } \\
\text { V } \\
\text { VI }\end{array}$ \\
\hline
\end{tabular}

El porcentaje de tráfico intrarregional está muy relacionado con la extensión de cada Región. Las Provincias suelen estar relacionadas con la capital importante más cercana y con las Provincias vecinas, por lo que, a medida que la extensión de la Región es mayor, ambos tipos de centros o núcleos están dentro de la misma Región y el tráfico intrarregional aumenta en consecuencia. Los valores máximos corresponden a Cataluña-Aragón, Galicia y la Meseta, y los mínimos a León-Oviedo.

El segundo porcentaje, relativo al tráfico interregional, depende de la extensión de las Regiones y, además, de la influencia de la actividad económica de la Región vecina más influyente. Los valores máximos corresponden a las Regiones antes citadas y a Andalucía occidental, y los mínimos también a León-Oviedo.

Puede afirmarse que, en líneas generales, hay una marcada relación entre la cifra de toneladas originadas y destinadas en cada

(*) I. Cataluña, Aragón, Soria y Castellón. II. Valencia, Alicante, Murcia y Albacete. III. Andalucía oriental. IV. Andalucía occidental. V. Galicia. VI. León y Oviedo. VII. Santander, Burgos, Vascongadas, Navarra y Logroño. VIII. La Meseta, a excepción de León, Burgos, Soria y Albacete. 
Provincia y su valor añadido bruto; que las Provincias agrarias especializadas en algún cultivo dan un saldo exportador, mientras que las de carácter industrial o de servicios son importadoras, y que un porcentaje importante del tráfico interprovincial de una Región permanece dentro de la zona delimitada por la misma más la Región vecina y Madrid.

Para el examen del tráfico en ferrocarril se dispone de la matriz origen-destino de las mercancías transportadas por RENFE en 1971, referidas a vagones completos, que suponen el 90 por 100 del total. De ella se deduce que las principales Provincias por el transporte originado son: Barcelona, Ciudad Real, Granada, León, Oviedo y Santander, y las principales Provincias receptoras son: Almería, Barcelona, Madrid, Oviedo y Pontevedra. Los saldos exportadores positivos se refieren a Provincias con predominio de producción minera o energética. 'En ambos sentidos, la Provincia más significativa es Oviedo, con un tráfico provincial del 75 por 100 , cuando el promedio es del 25.

La subdivisión de este tráfico, en las mismas Regiones consideradas con respecto al tráfico por carretera, permite afirmar que existen notables diferencias entre los porcentajes de origen y de destino; que en cuanto al tráfico intrarregional, los valores máximos en origen corresponden a Cataluña-Aragón y Galicia, y en destino, a León-Oviedo, Andalucía occidental y Cataluña-Aragón, y los mínimos, a Valencia-Murcia; y en cuanto al interregional, los máximos en origen corresponden a Cataluña-Aragón, Andalucía occidental y Galicia, y en destino, a Galicia y León-Oviedo, y los mínimos, también a Valencia-Murcia.

En resumen, puede afirmarse que el volumen de mercancías transportadas por tren en el tráfico intrarregional o interregional de recorridos cortos equivale a una quinta parte del tráfico total, absorbiendo la carretera los $4 / 5$, debido al menor coste, más favorecida en cuanto a inversiones públicas y que ofrece mejor servicio y la estructura de costes por ferrocarril en distancias cortas; pero que en recorridos largos, el tráfico intra o interprovincial es similar en carretera y en ferrocarril; que el aumento de la accesibilidad por mejora de las carreteras existentes aumentará tanto el tráfico intra como interprovincial, y que el ferrocarril, normalmente, ofrece costes menores que la carretera para gandes distancias y debería dedicarse más al tráfico interregional que al intrarregional o intraprovincial. 
Se desarrolla en la monografía que comentamos un extenso análisis de la competencia carretera-ferrocarril en el tráfico de mercancías y se hace un detenido examen de los servicios que se prestan, sus precios y tarifas según las cifras disponibles, en especial deducidas de encuestas específicas, por las que se llega a la conclusión de que los ferrocarriles se enfrentan con un sector altamente competitivo, cual es el de la carretera, que, por sus largas distancias, es más apropiado que el ferrocarril. Los precios de transporte por carretera están más ajustados a los costos y son francamente competitivos con los del ferrocarril. Los usuarios conceden mucha importancia a la calidad del servicio en el caso de la carretera y tienen muy en cuenta los precios en cuanto al ferrocarril se refiere.

Como consecuencia de este estudio se hacen unas estimaciones del tráfico de mercancias para 1981, cuyo límite superior en la hipótesis optimista es como sigue, en porcentajes:

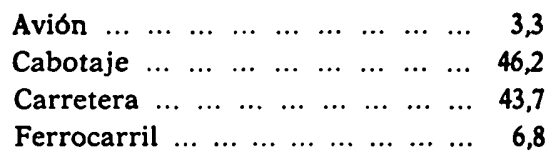

subrayándose la importancia mucho mayor de la carretera sobre el ferrocarril en el transporte de mercancías, aunque se sitúe un peldaño más abajo del transporte marítimo de cabotaje.

El tráfico originado en carretera para 1980, por Provincias, en porcentajes, es como sigue:

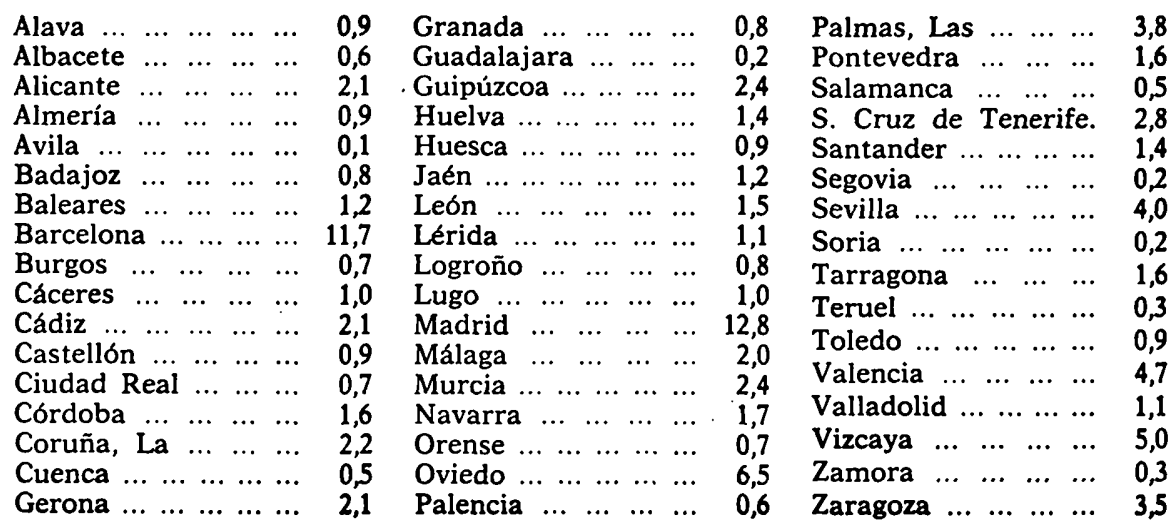


Destacan Madrid y Barcelona, que entre las dos absorben el 24,5 por 100 del tráfico total. En segundo lugar figuran Oviedo y Vizcaya, Valencia y Sevilla, Las Palmas y Zaragoza, cuyo tráfico, en conjunto, se eleva al 27,5 por 100 . Tan sólo ocho Provincias absorben más de la mitad del tráfico de mercancías, y si a ellas añadimos el tráfico de otras ocho Provincias - Alicante, Cádiz, La Coruna, Gerona, Guipúzcoa, Málaga y Murcia y Santa Cruz de Tenerife-, que representa el 18,1 por 100 , entre las 16 Provincias absorben más de $2 / 3$ de dicho tráfico.

\section{EL TRANSPORTE DE VIAJEROS}

La estructura del tráfico de viajeros ha cambiado sustancialmente en las últimas décadas, según se deduce de los siguientes porcentajes sobre las cifras totales de pasajeros-km.

\begin{tabular}{|c|c|c|c|}
\hline & 1955 & 1970 & 1980 \\
\hline $\begin{array}{llllllll}\text { Avión } & \ldots & \ldots & \ldots & \ldots & \ldots & \ldots & \ldots\end{array}$ & 1,13 & 1,94 & 2,90 \\
\hline Cabotaje $\ldots \ldots \ldots \ldots$ & 0,98 & 0,54 & 0,70 \\
\hline $\begin{array}{lllllll}\text { Carretera } & \ldots & \ldots & \ldots & \ldots & \ldots & \ldots\end{array}$ & 47,36 & 82,72 & 85,90 \\
\hline $\begin{array}{lllllll}\text { Ferrocarril } & \ldots & \ldots & \ldots & \ldots & \ldots\end{array}$ & 50,53 & 14,80 & 10,50 \\
\hline
\end{tabular}

Las cifras anteriores prueban que el transporte de viajeros por carretera, inferior del de transporte por ferrocarril en 1955, ha adquirido cada vez mayor importancia, mientras que ha disminuido progresivamente la importancia del ferrocarril, que en 1970 presenta un volumen de tráfico más de tres veces inferior al de 1955 y aún se prevé que prosiga la disminución hacia 1980.

Factor decisivo de este fenómeno ha sido el extraordinario incremento del parque de turismos, que, cifrado en 807.000 unidades en 1965, supera los tres millones en 1972, calculándose sus disponibilidades para 1980 en 8,8 millones. Es el automóvil privado el que más influye en este aumento del tráfico, ya que, aunque ha aumentado el parque de autobuses, el transporte colectivo en carretera tiene menor impacto.

El incremento del parque de turismos será en el futuro creciente en cifras absolutas, pero decreciente en los valores relativos expresivos de su ritmo de incremento. Las matriculaciones depen- 
derán más de la renovación del parque que de la difusión del automóvil.

En la tabla anterior se advierte un incremento persistente del tráfico aéreo y una situación desequilibrada y decreciente del tráfico de cabotaje, cuyo peso, en conjunto, es muy reducido en el tráfico total.

La previsión de la distribución provincial del tráfico de viajeros para 1980, calculada en función de la población y de la renta disponible por habitante, en cada Provincia, es como sigue:

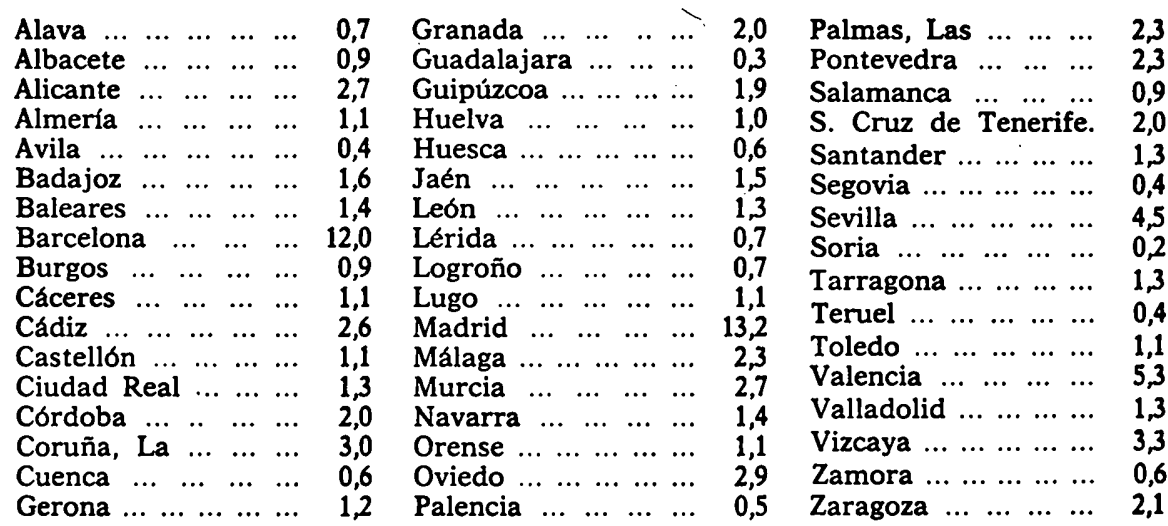

Las dos principales zonas de atracción de este tráfico de viajeros serán las Provincias y Areas metropolitanas de Barcelona y Madrid, que, al igual que con respecto al transporte de mercancías, suponen, en conjunto, la cuarta parte del tráfico nacional. En segundo término se sitúan, por orden de importancia, Valencia, Sevilla y Vizcaya, y en grado menor, La Coruña, Oviedo, Alicante, Murcia y Cádiz, cuyo tráfico, en conjunto, equivale a otra cuarta parte. Es decir, que la mitad del tráfico de pasajeros se concentrará en tan sólo 10 Provincias.

\section{INCIDENCIA DEL TRANSPORTE EN EL DESARROLLO REGIONAL}

\section{Principales Realizaciones}

Los autores del estudio afirman que la experiencia española acerca de la influencia del transporte sobre el desarrollo regional es escasa y señalan, como principales realizaciones de la Adminis- 
tración pública en este campo, los Planes de Badajoz y Jaén y las llevadas a cabo en los tres Planes de Desarrollo.

El Plan Badajoz tenía como objetivo la transformación agraria de las vegas del Guadiana y el fomento de la actividad industrial como complemento de aquélla. El 13 por 100 de las inversiones se aplican al transporte y a la construcción de $300 \mathrm{~km}$. de nuevas carreteras y 57 de vía férrea, con tramos de infra y superestructura en la línea Talavera de la Reina-Logrosán y al incremento del equipo de maniobra del pueblo de Huelva.

El Plan Jaén tenía por finalidad elevar el nivel medio de vida de la Provincia mediante la revalorización de sus recursos naturales, el proceso de industrialización, la mejora y ampliación de los regadíos, las repoblaciones forestales y el fomento de las explotaciones ganaderas. En el tramo ferroviario Baeza-Albacete se consumen el 20 por 100 de las inversiones totales.

La acción en el I Plan se concentra en la creación de siete polos de promoción o desarrollo en La Coruña, Vigo, Burgos, Valladolid, Zaragoza, Sevilla y Huelva, potenciales núcleos industriales, al objeto de disminuir el desempleo y la emigración de sus Provincias, en la Tierra de Campos y en el Campo de Gibraltar. Las inversiones en transporte se elevan en los polos al 25 por 100 de las totales y se aplican a las redes arteriales de carreteras y vías de acceso a los polos y en el ferrocarril Madrid-Burgos. En la Tierra de Campos se realiza por carretera y ferrocarril la mejora de las carreteras y la aplicación del Plan de Modernización de la RENFE en la Comarca. En el Campo de Gibraltar se mejoran las instalaciones portuarias y las carreteras de la Comarca. La atención al desarrollo urbano fue mínima.

La actuación en el II Plan se concreta en la descongestión de ciertas zonas, el impulso de las cabeceras de Comarca, la industrialización de Regiones con bajo nivel de renta y, como acción nueva, se tiende a la integración de la política regional y urbana y a incluir los sectores agrario y de servicios como materia de la intervención del Estado. Se crean cinco nuevos polos en Villagarcía de Arosa, Oviedo, Logroño, Granada y Córdoba. Las inversiones en transportes se elevan al 50 por 100 de las totales y se aplican a la construcción de ramales ferroviarios e.infraestructura de carreteras en los nuevos polos y a diversas acciones de descongestión de Areas metropolitanas y ordenación urbana y transportes marítimo y aéreo. 
En el III Plan se tiende a la planificación basada en ejes y no en polos, con un enfoque territorial de grandes áreas de expansión industrial. Las acciones fundamentales son: los programas regionales selectivos de Canarias, Galicia y el Sureste; las redes arteriales ferroviarias, armonizando la planificación urbana y metropolitana con los transportes urbanos y de cercanías; el esquema director de Madrid 2000, con acciones similares en Barcelona y Bilbao; la acción de la RENFE y las de carreteras y puertos.

La actuación de la RENFE se concreta en la red básica, con tráfico superior a $100 \mathrm{Tm} / \mathrm{km}$., y la complementaria, desde 4.000 a 10.000. Las inversiones se aplican en un 61 por 100 a la red básica, el 33 por 100 a la complementaria y un 5,6 por 100 a la secundaria para la construcción de estaciones de contenedores, implantación del servicio TECO entre Madrid y Barcelona con Bilbao y París, la terminación del ferrocarril Madrid-Burgos y la ampliación a vía ancha de los tramos Málaga-Fuengirola y Carcagente-Gandía. Se produce una desinversión, con cierre o desmantelamiento de líneas, atendiendo al criterio de que el ferrocarril no debe mantener servicios de débil tráfico que pueden ser prestados por la carretera, con menos consumo de recursos para la economía nacional, y se acentúan importantes reducciones de personal, de 122.400 agentes en 1963 a 90.400 en 1973.

En carreteras se lleva a ejecución el Plan de Red Primaria, de $13.300 \mathrm{~km}$., en el que se incluye el Plan Radial, de 5.000; las comunicaciones Asturias-León, y el Plan Nacional de Autopistas, incluidas las de Navarra y del Atlántico. Y en cuanto a puertos marítimos, la infra y superestructura de los de Villagarcía de Arosa y Bilbao.

\section{CONCLUSIONES}

En la parte IV de la monografía se analizan los modelos parciales de "localización», de "desarrollo urbano e interurbano" y el de "crecimiento regional», aplicados en otros países para el análisis de la interacción transporte-desarrollo regional, y se describe el nuevo modelo aplicado al caso español en el estudio que comentamos, las etapas consideradas en su aplicación y las variables que se tienen en cuenta - valor del suelo, población activa, tarifas, inversiones, tráfico de pasajeros y mercancías y congestión- y sus aspectos cualitativos y cuantitativos a largo, medio y corto plazo 
en los ámbitos nacional, provincial y en Areas metropolitanas, respectivamente. Igualmente se exponen los sistemas de evaluación beneficio/coste empleados por el Banco Mundial, la OCDE y las Naciones Unidas, así como los modelos de previsión, basados en el producto nacional bruto, la población activa y la elasticidad-renta del tráfico terrestre.

Fruto de este estudio, tendente a una ordenación racional del territorio a medio y largo plazo, con horizontes 1990, basado en el análisis beneficio/coste de las inversiones y en las encuestas de control del impacto del transporte, son los principios básicos de actuación en orden a la incidencia del transporte en el desarrollo regional, las conclusiones establecidas y previsiones formuladas cara al futuro.

Entre los principales principios básicos que deben tenerse en cuenta en la regulación del transporte figuran:

a) Los problemas del transporte no se resuelven sólo con inversiones, ya que existe una fuerte correlación entre producción agrícola e industrial, población activa industrial y de servicios, de un lado; tráfico generado - de mercancías y pasajeros-, de otro, e inversiones públicas efectuadas para absorber este tráfico.

b) Las inversiones planeadas y ejecutadas en función de los aumentos generan más tráfico urbano e interurbano, lo que permite afirmar que el tráfico sigue a las inversiones, y no al contrario.

c) Es conveniente adecuar el importe y localización de las inversiones en transporte a criterios de desarrollo espacial y no de rentabilidad privada, reconociendo la correspondencia existente entre transporte y desarrollo regional.

d) Una adecuada infraestructura del transporte es condición necesaria, aunque no suficiente del desarrollo. El desarrollo ha sido, en ocasiones, condicionante para promover las infraestructuras, como ocurrió en los comienzos del ferrocarril en Barcelona, Asturias y el País Vasco.

e) El desarrollo de una Provincia es función directa del índice de accesibilidad a las Provincias de mayor accesibilidad, lo que debe tenerse muy en cuenta en medidas de acción regional.

$f$ ) No puede hacerse una ordenación urbana sin una ordenación del transporte. El abandono de la ordenación del tráfico de pasajeros implica el abandono del control de su influencia sobre la ordenación urbana. 
Las principales conclusiones que se deducen de este estudio son:

a) La orientación radial y costera del tráfico terrestre, con tendencia a la concentración de los flujos en tres grandes ejes de dirección y atracción puede, en los próximos años, crear un desequilibrio regional importante al saturar las Areas metropolitanas de atracción, como Barcelona, Zaragoza y Bilbao, y en menor medida, Valencia y Asturias, esta última en el triángulo Oviedo-GijónAvilés, y habría que doblar la capacidad en los principales corredores de tráfico: Madrid-Zaragoza-Barcelona; Barcelona-La Junquera; Murcia-Alicante-Valencia-Barcelona; Vizcaya-Zaragoza-Barcelona, y Madrid-Burgos-Vizcaya-Irún, y exigiría el 21 por 100 de las inversiones públicas en el quinquenio 1985-1990.

Existen zonas especialmentes desfavorecidas en cuanto a su localización con respecto a Madrid, Barcelona y Vizcaya, como Andalucía occidental, Andalucía oriental y La Coruña.

b) La importancia de los ferrocarriles como aglutinadores de población ha disminuido en el período 1920-1970, frente a la creciente importancia de las carreteras y aeropuertos.

Los aeropuertos construidos donde más población había y más viajeros se generaban representan como aglutinadores de población y de actividad económica lo que el ferrocarril a principios de siglo.

Los puertos son tanto más importantes cuanto menos comunicaciones alternativas existen.

Las carreteras tienen una tendencia histórica similar a la de los aeropuertos, aunque no dependen tanto de medios alternativos, y sus beneficios parecen ser mayores cuanto mayor es la ciudad.

c) La política de modernización del ferrocarril desde 1962 no ha supuesto una alternativa viable al transporte por carretera debido a una falta de coordinación de las inversiones. Las inversiones del tráfico de carretera pueden significar en Regiones en proceso de desarrollo impactos decisivos para acelerar el aumento de producción y la concentración espacial.

Es de temer que el importe de las inversiones para provocar un despegue en Regiones subdesarrolladas deba ser superior a los beneficios obtenibles, por lo que es preferible incrementar el proceso de urbanización de ciudades de más de 50.000 habitantes que pueden acoger a la población activa de las zonas subdesarrolladas.

d) Existe un alto grado de enclavamiento del tráfico terrestre a nivel provincial y regional no económicamente adecuados para el 
transporte por ferrocarril en recorridos cortos. Este enclavamierito es tanto menor cuanto mayor es el desarrollo económico. Es conveniente un grado de desenclavamiento en forma de mejora de redes secundarias y de acceso. El transporte ferroviario, con recorridos medios de mercancías de $300 \mathrm{~km}$., no podrá constituir una alternativa al transporte por carretera hasta que el intercambio interregional e internacional conduzca a especializaciones territoriales sensibles y el recorrido medio supere los $400 \mathrm{~km}$., y ello depende de la industrialización española y de la incorporación de España a la CEE. Un esfuerzo comercial mediante camiones puerta-estación que capte el creciente tráfico interregional y la utilización de trenes rápidos de pasajeros son aconsejables en sumo grado.

e) Hay que evaluar los costes y beneficios del uso de la carretera, eliminando el tráfico que no cubra los costes marginales.

f) Los impactos territoriales, de no tomar medidas adecuadas, conducirán a que la concentración de la población se acelerara y, con ello, la concentración en el tráfico de pasajeros.

\section{Como previsiones cara al futuro se sugieren:}

a) Utilizar al máximo el ferrocarril en sus trayectos radiales.

b) Construcción de una red de grandes rutas concéntricas que unan entre sí las Provincias del centro del país, que debe conectarse a las rutas radiales existentes.

c) El Plan de Autopistas concéntricas -Atlántico, Cantábrico, Zaragoza-Mediterráneo, Mediterráneo- complementará las conexiones interregionales; pero hay que detener el Plan Nacional de Autopistas radiales, que competirá innecesariamente con las carreteras y ferrocarriles existentes, que tienen capacidad para absorber el tráfico económicamente justificado hasta 1985.

d) Descongestionar las zonas de accesos a Madrid, Barcelona y Bilbao, que influye sobre la estructura radial de las grandes carreteras nacionales. La solución consistiría, además de en una utilización intensiva del ferrocarril, en la construcción de centros de carga y descarga y de autopistas de circunvalación, cuyos vértices debieran estar situados a $60-80 \mathrm{~km}$. de la capital y que enlazarían las carreteras nacionales entre sí en puntos situados a gran distancia del Area metropolitana, evitándose los costes sociales de congestión y concentración urbana. 
e) Incrementar mediante subsidios cruzados entre ferrocarril y carretera las relaciones de tráfico interregional, pemitiendo a la carretera actuar como factor descentralizador de la actividad económica, mejorando las comunicaciones interregionales.

En forma sistemática exponen los autores, como resumen, la politica recomendada:
A) Grandes Areas metropolitanas

- Carreteras

- Construir centros periféricos de carga y descarga comunes con ferrocarril.

- Limitar transportes de mercancías por trayectos y líneas.

- Delimitar carriles especiales.

- Subvencionar transporte público y penalizar el privado.

- Construir cinturones de circunvalación a 60-80 km.

- Aumentar por medios fiscales la capacidad de los vehículos.

\section{- Ferrocarriles}

- Establecer servicios puerta a puerta con camión.

- Aumentar capacidad y velocidad de líneas radiales mediante telecomunicaciones.

- Subvencionar retornos en líneas radiales.

- Aeropuertos

- Situar aeropuertos a $60-80 \mathrm{~km}$. del centro y habilitar ac. cesos de masas.

\section{- Puertos}

- Aumentar la capacidad mediante mejora de operaciones o inversiones. 
B) Ciudades medias

- Carreteras

- Construir centros periféricos de carga y descarga comunes con ferrocarril.

- Limitar transporte de mercancias por trayectos y horas.

- Subvencionar el transporte público y penalizar el privado.

- Ferrocarriles

- Establecer servicios puerta a puerta con camión.

- Aumentar capacidad y velocidad de líneas radiales mediante telecomunicaciones.

- Subvencionar retornos en líneas radiales.

- Puertos

- Mejorar operaciones.

C) Zonas desarrolladas

- Carreteras

- Establecer peajes en carreteras radiales.

- Mejorar accesos interregionales.

- Ferrocarriles

- Subvencionar transportes radiales.

D) Zonas subdesarrolladas

- Mejorar carreteras de acceso. 


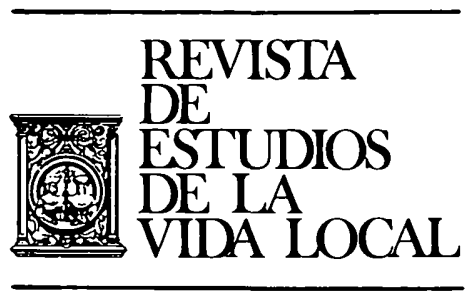

IV. JURISPRUDENCIA 
REVL-1978, núm. 197. BALLESTER ROS, IGNACIO. NOTAS SOBRE LA EVOLUCION Y ESTRUCTU... 